\title{
Early results of bidirectional cone sutures for mid-face lifting in Asian patients
}

\author{
Phoebe Kar Wai Lam, MBCHB, MRCS, MSCPD (D, James Yui Lam, MBBS, MRCSED, PGDIPCLINDERM(D, \\ Edwin Kwan Chark Lau, MBBS ID, Wang Lung Luk, MBBS, MSc, FCOHK, FCSHK iD
}

Association of Doctors in Aesthetic Medicine (ADAM), Hong Kong

\begin{abstract}
Background: There are intrinsic difference facial aesthetics between Asian and Caucasian, as well as racial differences in beauty standards. Bi-directional cones threads are effective in mid-face lifting but there was yet no study in Asians.

Objective: This study aimed to determine the effects on mid-face laxity the effect size of using bidirectional cone suture for facelifting in Asian patients.

Methods: In this prospective study, three pairs of bidirectional cone threads were used for mid-face lifting in 10 healthy volunteers with mild-to-moderate facial laxity.

Results: The validated assessment parameters used in the study were the facial laxity rating scale (FLRS) to evaluate the severity of mid-face laxity and the wrinkle severity rating scale (WSRS) to assess the severity of the nasolabial fold (NLF). Overall, there was a substantial improvement in mid-face laxity and the NLF after thread lifting, supported by treatment effect size (Kendall W's value: 0.96 and 0.85 , respectively). Significant improvements for both mid-face laxity and NLF were observed in all patients by 3 months $(p<0.05)$, with significant differences in FLRS $(p=0.000)$ and WSRS $(p=0.001)$. At each follow-up, all patients would complete a selfadministered questionnaire that included the subjective satisfaction rating scale. The results showed that patient satisfaction was substantially improved; the differences between baseline and the 6 -week and 3 -month follow-up were significant ( $p<0.05)$, and the treatment effect size was $W=0.950(p<0.05)$. All subjects tolerated the procedure well with transient and minimal side effects.

Conclusion: In Asians with mild-to-moderate laxity, absorbable cones sutures produced considerable improvement in mid-face laxity and NLF within three months. The findings were consistent among the three reviewers and were statistically significant $(p<0.05)$. Patient satisfaction was higher at the 6-week and 3-month follow-up compared to the baseline $(p<0.05)$.
\end{abstract}

Keywords: fascia; ligament and aging; nasolabial fold; rhydioplasty

\section{Introduction}

The skin of Asians has a thicker dermal layer [1] with abundant collagen and melanin. It is, therefore, less susceptible to the development of wrinkles, rhytids, and photoaging compared to that of Caucasians. However, Asians are more predisposed to the sagging of soft tissue with age than Caucasians as thicker and heavier skin is accompanied by weaker facial skeletal support, a flatter mid-face, more substantial soft tissue, and a weaker chin. Therefore, when repositioning descended soft tissues in Asians, it is essential to provide a more durable lift with good anchorage that can withstand the repetitive opposing dynamic forces involved in daily facial movements.

The superficial fat compartments are tightly adherent to the

Received May 4, 2020; Revised May 22, 2020; Accepted May 26, 2020

Corresponding author: Phoebe Kar Wai Lam

E-mail: myperfectskinsolution@gmail.com

This is an Open Access article distributed under the terms of the Creative Commons Attribution Non-Commercial License (http://creativecommons.org/licenses/by-nc/4.0), which permits unrestricted non-commercial use, distribution, and reproduction in any medium, provided the original work is properly cited.

Copyright $@ 2020$ Korean Society of Korean Cosmetic Surgery and Medicine (KSKCS \& KCCS). 
overlying skin and restricted by the facial retaining ligaments. While the volume of the superficial fat pad remains relatively constant over time, that of the deep fat pad progressively reduces. The loss of structural mid-face support and the progressively increasing ligament laxity, together with the restrictive descent of superficial fat tissues, resulting in the distinctive signs of midface ageing - nasolabial folds (NLFs) and marionette lines [2-4].

The composite material of the absorbable cone suture is polyglycolide/L-lactide (PLGA). The US Food and Drug Administration (FDA) approved the use of absorbable bidirectional cone thread sutures for mid-face lifting. The bidirectional cone sutures reposition the sagging superficial fat pads and skin through diverse mechanisms that occur over time. First, the bidirectional threads act as a scaffold that lifts the descended soft tissue. Second, the threads stabilise the repositioned tissues within the facial retaining ligaments. Third, delayed collagen stimulation and regeneration continue to maintain the ptotic mass in its new position even after absorption of the suture threads.

The investigation is a 24-month prospective study that evaluates the mid-face lifting outcome of bidirectional cone sutures in Asians with mild-to-moderate facial laxity. This current article described the results comparing baseline, six weeks and three months follow-up.

\section{Materials and methods}

The inclusion criteria included healthy volunteers with mildto-moderate facial laxity according to the facial laxity rating scale (FLRS) without any history of aesthetic procedures of the mid-face. A total of 10 volunteers aged 24 to 53 years (average, 42.4 years) recruited into the study between September 2019 and January 2020. Assessments for both mid-face laxity and the NLF using the FLRS the wrinkle severity rating scale (WSRS), respectively, were validated by three independent reviewers. Improvement is defined as a change in at least one grade from the baseline on the FLRS. Self-administered satisfaction questionnaires, including the global aesthetic improvement scale (GAIS) and the individual subjective satisfaction rating scale (SSRS), were completed by the participants during each follow-up visit.

\section{Procedure}

Effective vectors refer to the directions of straight-line forces that directly reposition the targeted treatment area. The last cone of each distal suture should be anchored within the superficial fat pad to produce the most efficient pulling point (EPP).
The entry and exit points (about $6 \mathrm{~cm}$ and $12 \mathrm{~cm}$ proximal to the EPP, respectively) were pre-marked in the sitting position.

After sterilisation of the face, I at entry injected $0.2-0.3 \mathrm{ml}$ of Xylestesin-A (1:80,000; 3M ESPS, Germany) at each pre-marked entry and exit points and I punctured the skin with an 18-G needle. The double-arm sutures passed perpendicularly piercing through the surface till they reached the 5-mm mark. The needle was then pivoted into and continued along the subdermal plane. The two needles were transferred in opposite directions (proximally and distally) along the direction parallel to the desired vector of movement. I gently massaged the soft tissue over the cones of the sutures until the needles entirely passed through the skin via the exit points. Once the desired effect is achieved, any excess length of suture was trimmed.

Three pairs of absorbable sutures inserted in straight parallel vectors in the mid-face for each participant.

Clinical two-dimensional photographs were taken using the Nikon D5200 (Tokyo, Japan), and three-dimensional digital photos using the Mirage system were taken at the 6-week and 3-month follow-ups and 6-, 9-, 12-, and 24-month follow-up.

Statistical analyses performed aimed to compare the follow-up results of both the reviewers and participants against the baseline data. The chi-squared test used to analyse the change in facial laxity, NLF, and patient satisfaction (SSRS and GAIS) at a 95\% confidence level. The Kendell's W test determines the effect size of thread lifting on the participants before and after treatment.

\section{Results}

The current study reports the resultsrecorded at baseline and the 6-week and 3-month follow-ups. Overall, there were observable differences following thread-lift insertion over time (Fig. 1, 2).

\section{Facial laxity rating scale}

An improvement (an enhancement of at least one grade on the assessment scale) in FLRS was observed in 70\%-90\% patients and up to $100 \%$ patients by three months (Table 1 ). The treatment effect size was large $(\mathrm{W}=0.96)$. Among the results collected by the three reviewers, at least one of the periods significantly differed $\left(\chi^{2}(2)=19.2 ; \mathrm{p}=0.000\right)$. All the three reviewers found significant FLRS improvement between baseline and the 3-month follow-up ( $\mathrm{p}=0.000)$. Two reviewers (RII and RIII) found significant FLRS improvement between the 6-week and the 3-months periods ( $\mathrm{p}=0.030$; Table 2). 


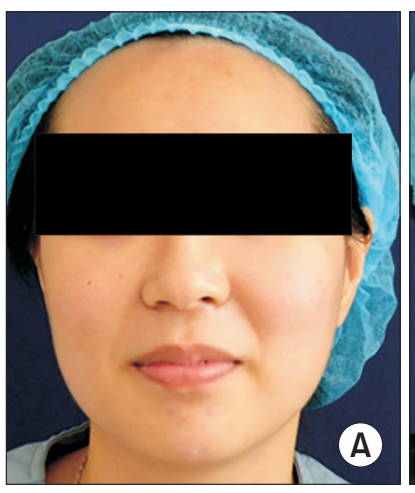

Baseline

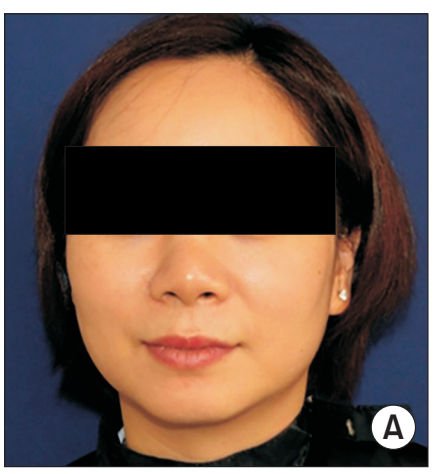

Baseline

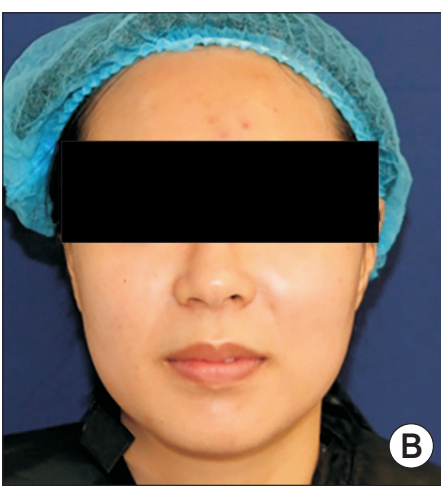

6 weeks

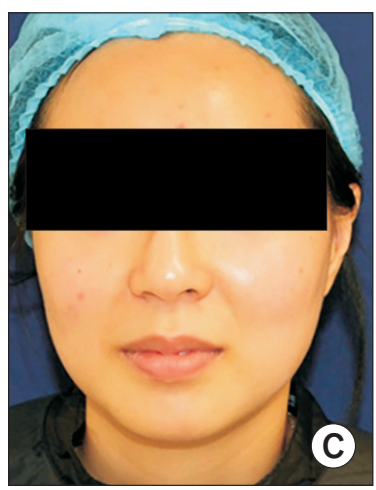

3 months

1. Patient one, pictures taken at baseline (A) and follow-up at 6 weeks (B) and 3 months (C).

Table 1. Proportion of patients showing improvement in the facial laxity rating scale (FLRS) at follow-up

\begin{tabular}{ccc}
\hline FLRS & No improvement (\%) & Improvement (\%) \\
\hline 6 weeks & $10-30$ & $70-90$ \\
3 months & 0 & 100 \\
\hline
\end{tabular}

No improvement means no change in FLRS grading scale between the follow-up interval ( 6 weeks or 3 months) compared to baseline. Improvement refers to any change (i.e. [greater than or equal to]) 1 change in FLRS grading scale between follow-up interval and baseline.

\section{Wrinkle severity rating scale}

At the 6-week follow-up, $50.0 \%$ of patients showed an improvement in the NLF, and all patients showed improvement at the 3-month follow-up (Table 3). The treatment effects were significant $(\mathrm{W}=0.85)$. At least one of the periods were significantly different $\left(\chi^{2}(2)=16.9 ; p=0.000\right)$. Similar to FLRS, all three reviewers found significant WSRS improvement between baseline and the 3-month follow-up ( $\mathrm{p}=0.000-0.001$ ), and two reviewers (RII and RIII) found significant improvement between the 6-week and the 3-month periods ( $\mathrm{p}=0.0145$; Table 4 ).
Table 2. Proportion of patients showing improvement in the wrinkle severity rating scale (WSRS) at follow-up

\begin{tabular}{lrrr}
\hline \multicolumn{1}{c}{ FLRS } & RI & RII & RIII \\
\hline p-value & 0.000 & 0.000 & 0.000 \\
$\chi^{2}(2)$ & 19.538 & 18.865 & 18.865 \\
Kendall's W & 0.977 & 0.943 & 0.943 \\
Adjusted significance & & & \\
Baseline-6 wk & 1.33 & 0.353 & 0.353 \\
6 wk-3 mo & 0.057 & 0.030 & 0.030 \\
Baseline-3 mo & 0.000 & 0.000 & 0.000 \\
\hline
\end{tabular}

No improvement means no change in WSRS in grading scale between the follow-up interval (6 weeks or 3 months) compared to baseline. Improvement refers to any change (i.e. [greater than or equal to]) 1 change in the facial laxity rating scale (FLRS) grading scale between follow-up interval and baseline.

\section{Subjective satisfaction (the global aesthetic} improvement scale and the subjective satisfaction rating scale)

Treatment effects on GAIS was moderate $(\mathrm{W}=0.600)$. The difference between the 6-week and 3-month follow-up was not significant $(\mathrm{p}<0.005)$. However, the treatment effects on SSRS was substantial $(\mathrm{W}=0.950 ; \mathrm{p}<0.05)$. There were significant dif- 
Table 3. A chi-squared determines how much difference between WSRS assessment rating and treatment effect (Kendall's W) at 6 weeks and 3 months in the 3 reviewers (RI, RII, RIII)

\begin{tabular}{ccc}
\hline WSRS & No improvement (\%) & Improvement (\%) \\
\hline 6 weeks & 50.0 & 50.0 \\
3 months & 0 & 100 \\
\hline
\end{tabular}

WSRS, the wrinkle severity rating scale.

Table 4. Statistical analysis on the difference between WSRS assessment rating and treatment effect (Kendall's W) at 6 weeks and 3 months in the 3 reviewers (RI, RII, RIII)

\begin{tabular}{lrrr}
\hline \multicolumn{1}{c}{ WSRS } & \multicolumn{1}{c}{ RI } & \multicolumn{1}{c}{ RII } & \multicolumn{1}{c}{ RIII } \\
\hline p-value & 0.000 & 0.000 & 0.000 \\
$\chi^{2}(2)$ & 15.235 & 18.588 & 18.571 \\
Kendall's W & 0.762 & 0.929 & 0.929 \\
Adjusted significance & & & \\
Baseline-6 wk & 0.438 & $<0.999$ & 0.791 \\
6 wk-3 mo & 0.101 & 0.011 & 0.016 \\
Baseline-3 mo & 0.001 & 0.000 & 0.000 \\
\hline
\end{tabular}

WSRS, the wrinkle severity rating scale.

ferences in subjective rating between baseline and the 6-weeks and 3-month follow-ups ( $\mathrm{p}<0.05$; Table 5,6 ). All subjects tolerated the procedure well with transient and minimal side effects.

\section{Discussion}

Facial retaining ligaments anchor the tissue layers to the underlying skeleton, and spaces between these ligaments are soft tissue planes that allow gliding movements of the superficial fascia over the deep fascia. This ligamental system and the immobile fascia are secure networks on which lifting sutures can be anchored, naturally reversing the ageing features of descending soft tissue over their boundaries with the loss of tissue support [5-7].

In terms of suture placement, the threads cross the retaining ligaments with the proximal part of the lifting-threads anchored within the immobile fascia, and the distal cones attached to the targeted superficial fat pads to produce the EPP. Consequently, natural lifting and repositioning of the descended tissue will be stable and substantial. As established in previous literature, sutures should be placed as straight-line vector planning and inserted in the direction perpendicular to the target plane to achieve the most effective vector for elevation. The extent of lifting will, therefore, proportionally increase with the total number of sutures placed in a stackable approach [8].

Our results in treating mild-to-moderate mid-face laxity in Asian subjects with bidirectional cone sutures were consistent
Table 5. The treatment effect size upon SSRS and GAIS in 10 study subjects

\begin{tabular}{lcc}
\hline \multicolumn{1}{c}{ Variable } & SSRS & GAIS \\
\hline Kendall's W & 0.600 & 0.950 \\
Test statistic (n) & 12 & 19 \\
D.F. & 2 & 2 \\
Asymptotic significance (2-sided) & 0.002 & 0.000 \\
\hline
\end{tabular}

SSRS, the subjective satisfaction rating scale; GAIS, the global aesthetic improvement scale; D.F., degree of freedom.

Table 6. Difference between the scored SSRS and GAIS at different time-interval in the 10 study subjects

\begin{tabular}{lcccc}
\hline \multicolumn{1}{c}{ Variable } & Test stat & SE & Significance & Adj significance \\
\hline SSRS & & & & \\
Baseline-6 wk & 0.9 & 0.447 & 0.044 & 0.133 \\
Baseline-3 mo & 0.9 & 0.447 & 0.044 & 0.133 \\
GAIS & & & & \\
Basline-6 wk & -1.4 & 0.447 & -3.13 & 0.005 \\
Baseline-3 mo & -1.6 & 0.447 & -3.57 & 0.001 \\
\hline
\end{tabular}

SSRS, the subjective satisfaction rating scale; GAIS, the global aesthetic improvement scale; stat, statistic; SE, standard error; Adj, adjusted.

with those of previous studies. Perceivable changes in mid-face laxity and the NLF were detected as early as six weeks, although all three reviewers verified significant differences $(\mathrm{p}<0.05)$ only at three months after the procedure.

The time frame for observable clinical improvement usually correlates with the time required for the formation of new collagen that acts as a scaffold for the newly positioned tissue. An enhanced mid-face volume can, therefore, be achieved between 6 weeks to 3 months.

The overall duration of marked improvement may potentially last up to 36 months, secondary to the composite material PLGA in sutures that stimulates new collagen production [9]. Future studies should investigate whether the complimentary use of growth factors, cytokines, vitamins, or zinc may further enhance collagen synthesis, which may, in turn, amplify the treatment effects and shorten the duration for observable results.

Our mid-to-long term (24-month) assessment analysis will be available next year, and an update on treatment effects and patient satisfaction will be reported. One of the major limiting factors for our current prospective study is the small number of study participants. A multi-centre study involving a more significant participant sample size would be beneficial. 


\section{Conflicts of interest}

This is a sponsored drug study. All the study material are produced for by Clover Medical Technology (Hong Kong Limited Ltd.).

\section{References}

1. Park DM. Total facelift: forehead lift, midface lift, and neck lift. Arch Plast Surg 2015;42:111-25.

2. Yousif NJ, Matloub H, Summers AN. The midface sling: a new technique to rejuvenate the midface. Plast Reconstr Surg 2002;110:1541-53.

3. Rohrich RJ, Pessa JE. The fat compartments of the face: anatomy and clinical implications for cosmetic surgery. Plast Reconstr Surg 2007;119:2219-27.

4. Dumont T, Simon E, Stricker M, Khan JL, Chassagne JF. [Analysis of the implications of the adipose tissue in facial morphology, from a review of the literature and dissections of
10 half-faces]. Ann Chir Plast Esthet 2007;52:196-205. French.

5. Mendelson BC, Jacobson SR. Surgical anatomy of the midcheek: facial layers, spaces, and the midcheek segments. Clin Plast Surg 2008;35:395-404; discussion 393.

6. LaTrenta G. The aesthetic anatomy of the face. In: LaTrenta G, editor. Atlas of aesthetic face \& neck surgery. Philadelphia, PA: Saunders; 2004. p. 2-44.

7. Lorenc ZP, Goldberg D, Nestor M. Straight-line vector planning for optimal results with silhouette instalift in minimally invasive tissue repositioning for facial rejuvenation. J Drugs Dermatol 2018;17:786-93.

8. Goldberg D, Guana A, Volk A, Daro-Kaftan E. Single-arm study for the characterization of human tissue response to injectable poly-L-lactic acid. Dermatol Surg 2013;39:915-22.

9. Ogilvie MP, Few JW Jr, Tomur SS, Teven CM, Semersky AJ, Bruno CR, et al. Rejuvenating the face: an analysis of $100 \mathrm{ab}-$ sorbable suture suspension patients. Aesthet Surg J 2018;38: 654-63. 